\title{
El sobreendeudamiento y su impacto en el riesgo de crédito en instituciones microfinancieras peruanas: cajas municipales de ahorro y crédito 2016-2019
}

\section{Emerson Jesús Toledo Concha, Víctor Manuel León Reyes}

Departamento Académico de Ciencias Contables, Pontificia Universidad Católica del Perú

Consultor independiente

La micro y la pequeña empresa en Perú se encuentra en constante desarrollo desde hace cuarenta años. Desde entonces, aparecieron las cajas municipales de ahorro y crédito (CMAC), que tienen autonomía administrativa, financiera y económica, y participan de la descentralización de los servicios financieros. Sin embargo, el crecimiento de la cartera de colocaciones viene acompañado de elevados indicadores de morosidad relacionados al riesgo de crédito de estas instituciones. El objetivo del presente trabajo es analizar si el sobreendeudamiento de clientes, medido como el saldo y número de entidades financieras que tiene el deudor, es una variable que tiene una relación positiva y directa al riesgo de crédito, lo que lleva a constituir mayores provisiones que inciden negativamente en los estados de los resultados de este grupo de instituciones. Esta investigación es de enfoque cuantitativo y correlacional. Los datos recolectados de 48 meses (2016-2019) fueron procesados utilizando la metodología estadística CRISP-DM. Como resultado, se determinó la relación directa del sobreendeudamiento con el riesgo de crédito de las CMAC. La relevancia de esta investigación está dirigida a tomar decisiones estratégicas que permitan tener un mejor control del comportamiento de pago de sus operaciones de crédito y que esto se vea reflejado en mejores resultados económicos.

Palabras clave: microfinanzas, sobreendeudamiento, riesgo de crédito, cajas municipales

\section{Over-indebtedness and its impact on credit risk in Peruvian microfinance institutions: cajas municipales de aborro y crédito 2016-2019}

The micro and small business in Peru has been in constant development for 40 years, since then the Municipal Savings and Credit Banks (CMAC) appeared, which have administrative, financial, and economic autonomy and participate in the decentralization of the financial services; However, the growth of the loan portfolio is accompanied by high delinquency indicators related to the credit risk of these institutions. The objective of this work is to analyze whether the over-indebtedness of clients, measured as the balance and number of financial entities that the debtor has, is a variable that has a positive and direct relationship to credit risk, leading to the establishment of higher provisions that negatively affect in the income statements of this group of institutions. This research has a quantitative and correlational approach, where the data collected from 48 months, (2016-2019), were processed using the statistical methodology CRISP-DM, where the direct relationship of the over-indebtedness with the credit risk of the CMAC. The relevance of this research is aimed at making strategic decisions that allow better control of the payment behavior of your credit operations and this is reflected in better economic results.

Keywords: microfinance, over-indebtedness, credit risk, municipal savings banks 


\section{Superendividamento e seu impacto sobre o risco de crédito em instituiçóes de microfinanças peruanas: cajas municipales de aborro y crédito 2016-2019}

A micro e pequena empresa no Peru está em constante desenvolvimento há 40 anos, desde então surgiram os Bancos Municipais de Poupança e Crédito (CMAC), que possuem autonomia administrativa, financeira e econômica e participam da descentralização dos serviços financeiros; No entanto, o crescimento da carteira de crédito é acompanhado por elevados indicadores de inadimplência relacionados ao risco de crédito dessas instituiçóes. O objetivo deste trabalho é analisar se o superendividamento de clientes, medido pelo saldo e número de entidades financeiras que o devedor possui, é uma variável que tem relaçáo direta e positiva com o risco de crédito, levando ao estabelecimento de maiores disposiçôes que afetam negativamente as demonstraçôes de resultados deste grupo de instituiçôes. Esta pesquisa possui uma abordagem quantitativa e correlacional, onde os dados coletados de 48 meses, (2016-2019), foram processados utilizando a metodologia estatística CRISP-DM, onde a relação direta do superendividamento com o risco de crédito do CMAC é encontrado. A relevância desta pesquisa está voltada para a tomada de decisóes estratégicas que permitam melhor controle do comportamento de pagamento de suas operaçôes de crédito e isso se reflita em melhores resultados econômicos.

Palavras-chave: microfinanças, superendividamento, risco de crédito, caixa econômica municipal

\section{Introducción}

El desarrollo de las microfinanzas en Perú ha tenido un crecimiento constante, debido al inmenso potencial de desarrollo en la atención de este mercado emergente, aunque existan millones de personas que se encuentran desatendidas por los diversos sistemas financieros. Sin embargo, ahora se otorgan préstamos a personas emprendedoras de bajos recursos sin solicitar garantías: solo se pide una garantía solidaria. Actualmente, las instituciones financieras especializadas ofrecen servicios financieros, como capital de trabajo, compra de activos fijos, o para el consumo de los microempresarios, la refacción de sus viviendas, el envío de remesas, diversos tipos de cuentas de ahorros o microseguros; inclusive, cuentan con sus servicios de banca electrónica. Las instituciones realizan un gran aporte al desarrollo financiero, sociocultural y socioeconómico, como señala Pereira (2019):
Las pequeñas y medianas empresas, a nivel mundial, se han convertido en tema relevante en la actualidad económica para muchos países, los cuales generan interés en la creación y fortalecimiento de empresas cada vez competitivas como motor de desarrollo de sus gobiernos (p. 90).

Por otro lado, de acuerdo a los datos estadísticos macroeconómicos presentados por Mipyme, oficina de estudios perteneciente al Ministerio de la Producción $(2020)^{1}$, el 96,04\% de las empresas en Perú corresponden a la micro y pequeña empresa $(88 \%$ en el sector comercio y servicios, y $12 \%$ en la actividad productiva). Además, dicho grupo forma parte del $60 \%$ del total de la población económicamente activa (PEA) y del 40\% del producto bruto interno (PBI).

Las instituciones dedicadas al sector de la micro y pequeña empresa en Perú iniciaron sus actividades en los años ochenta. Desde entonces, fueron surgiendo

1 El Ministerio de la Producción es un órgano del Poder Ejecutivo en Perú, que se encarga de formular, aprobar, ejecutar y supervisar los niveles de producción, industria, manufacturera y pesquera. 
empresas más consolidadas, con mayor nivel patrimonial y participación de los gobiernos municipales de cada región. Esto propició un buen clima para la atracción de capitales de fondos extranjeros especializados en el otorgamiento de créditos al sector. Así, se crearon las cajas municipales de ahorro y crédito (en adelante, CMAC), que se rigen de acuerdo con la Ley 26702 (Ley General del Sistema Financiero y del Sistema de Seguros y Orgánica de la Superintendencia de Banca y Seguros). Las CMAC son instituciones financieras reguladas por el Estado y pertenecen a los gobiernos municipales de cada provincia; además, reciben depósitos y se especializan en el otorgamiento de créditos a la micro y pequeña empresa.

El objetivo del presente trabajo es analizar el sobreendeudamiento de los clientes a partir del riesgo de crédito de las CMAC; es decir, se busca indagar si existe una relación directa y positiva con respecto a este tipo de riesgo. Hasta la fecha, el crecimiento de dichas instituciones ha ido de la mano de indicadores de morosidad con tendencia creciente que afectan los resultados de las CMAC. Esos indicadores son consecuencia del comportamiento de diversos factores que afectan o inciden en el riesgo de crédito, que en este trabajo es entendido como la cartera de alto riesgo. $\mathrm{Al}$ mismo tiempo, cuando los clientes logran acceder a préstamos en diversas entidades, no pueden cumplir con sus obligaciones de pago; es decir, mientras mayor es el saldo y el número de entidades del deudor, mayores serán sus dificultades de pago, ya sea por su menor capacidad financiera o por su voluntad de cumplir con la institución. Esto conlleva a que las CMAC incrementen su nivel de morosidad y el monto de sus provisiones, lo que afecta directamente sus resultados.

Los resultados de esta investigación contribuirán al desarrollo de nuevos juicios y elementos de análisis que permitan entender mejor el comportamiento del riesgo de crédito de este grupo de instituciones. De esta manera, se podrán tomar mejores decisiones estratégicas y, por ende, se podrá mejorar la gestión de riesgos.

\section{Revisión bibliográfica}

Es importante revisar la definición de micro y pequeña empresa, en vista que son el mercado objetivo de atención de las CMAC. Cabe precisar que existen diversos criterios y metodologías de clasificación para la micro y pequeña empresa en el mundo. Para esta investigación, se utilizará la definición establecida en la legislación peruana. En Perú, la Ley 28015 (Ley de la Promoción y Formalización de la Micro y Pequeña Empresa) establece lo siguiente:

La micro y pequeña empresa es la unidad económica constituida por una persona natural o jurídica, bajo cualquier forma de organización o gestión empresarial contemplada en la legislación vigente, tiene por objeto desarrollar diversas actividades entre las cuales pueden ser la extracción, transformación, producción, comercialización de bienes o prestación de servicios (2003, art. 2).

Asimismo, la Superintendencia de Banca, Seguros y AFP (en adelante, SBS) establece que, en el sistema financiero, la micro y pequeńa empresa está regida de la siguiente manera:

- Microempresas: personas naturales o jurídicas, cuyo nivel de endeudamiento en el sistema financiero es menor a 20.000 soles en los últimos seis meses

- Pequeña empresa: personas naturales o jurídicas, cuyo nivel de endeudamiento es mayor a 20.000 soles y menor a 300.000 soles (Resolución SBS 11356-2008) 
En Perú, se fomenta el desarrollo de la micro y pequeña empresa, apoyando la mejora de su productividad, competitividad y la formalización. Estas medidas tienen un impacto en un empleo sostenible y en el producto bruto interno del país.

Ledgerwood (2000) señala que los riesgos de crédito en microfinanzas desempeñan un papel importante en los flujos de efectivo, gastos y rentabilidad de las instituciones. Asimismo, indica que la calidad de la cartera depende de muchos factores, como la administración en el número de clientes (número de préstamos activos por oficial de crédito), el crecimiento desmedido de la cartera de créditos, políticas crediticias y el incremento del monto promedio de las operaciones; este último factor está relacionado a un mayor saldo de préstamo por deudor en promedio.

Por otro lado, el control de la calidad de la cartera es fundamental en las instituciones microfinancieras. Vela, Uriol, Medina, Palacios y Pintado (2012) hacen referencia a que los préstamos morosos desempeñan roles críticos en los gastos, ya que afectan los flujos de efectivo, los ingresos y la rentabilidad de las instituciones microfinancieras. Mientras más esfuerzos y recursos se empleen en la recuperación de los créditos, se tendrán menos recursos disponibles para poder atender las operaciones. Además, los efectos más significativos se dan cuando no se paga el capital del préstamo, pues las instituciones financieras tienen que generar provisiones, que afectan directamente al gasto en el estado de ganancias y pérdidas. Por ello, las cuentas patrimoniales y las cuentas de resultados se ven afectadas:

En las instituciones microfinancieras los préstamos no están generalmente respaldados por una garantía realizable, de modo que la calidad de la cartera es el indicador más importante, para la sostenibilidad a largo plazo porque la mayor fuente de riesgo está en sus carteras de créditos (Vela et al., 2012, p. 9).
Frente a los diferentes riesgos posibles, Aguilar, Camargo y Morales (2004) definen el «riesgo de crédito». Se trata del caso de riesgo «más importante al que debe hacer frente cualquier entidad financiera. Un indicador del riesgo crediticio es el nivel de morosidad de la entidad, es decir, la proporción de su cartera que se encuentra en calidad de incumplimiento» (p. 3). Además, los autores señalan que un indicador del riesgo de crédito es el nivel de morosidad, que consiste en los créditos con atrasos en sus pagos. Por ello, en el presente artículo, la variable dependiente es definida por el riesgo de crédito, que está determinado por una tasa de morosidad, indicador importante para conocer la calidad de la cartera de las instituciones.

Con respecto a la variable independiente, que es el sobreendeudamiento de los clientes, se buscará demostrar que tiene relación directa con el riesgo de crédito. De acuerdo con Adrián González y Claudio Gonzáles-Vega (2003), «el sobreendeudamiento es el surgimiento de dificultades de pago que pueden resultar de la falta de voluntad de pago, insuficiente capacidad de pago o necesidad de incurrir en acciones costosas para poder pagar» (p. 13). Por su parte, Rossi (2017) define el «sobreendeudamiento» como «la situación de imposibilidad cierta, actual o futura de cumplir con las obligaciones generadas en relaciones de consumo en la que se encuentra un cliente de buena fe» (p. 92). Asimismo, con respecto a los problemas de sobreendeudamiento que están teniendo los microempresarios y el papel importante que deben de tener las instituciones microfinancieras en el control del mismo, Schicks (2010) menciona lo siguiente:

El sobreendeudamiento, es un grave riesgo para la industria de las microfinanzas. Primero, contradice completamente la misión social del sector. Puede mejorar la pobreza de los clientes de microfinanzas, amenazar sus redes sociales, posición e incluso conducir a tras- 
tornos psicológicos y problemas de salud. Segundo, a más tardar cuando el sobreendeudamiento desencadena el incumplimiento, representa una amenaza para la sostenibilidad institucional de las instituciones microfinancieras. Como tercer aspecto, el sobreendeudamiento amenaza con dañar la reputación de las microfinanzas, industria como tal que tienen efectos indirectos que afectarían incluso a instituciones saludables y sus clientes [traducción propia] (p.24).

El sobreendeudamiento es producto de la competencia (cada vez más intensa) de instituciones en el otorgamiento de créditos a este sector. Así, las entidades toman estrategias agresivas en la compra de deudas para despojarse de clientes. De acuerdo con lo mencionado por García (2019) en Gestión, que es uno de los diarios importantes de Lima, los bancos y financieras que dan microcréditos ofrecen tasas de interés cada vez más bajas en la actualidad. De esta manera, las entidades financieras tratan de ganar mayor participación de mercado, muchas veces a costa de los clientes.

Goldberg y Palladini (2011) señalan que las instituciones deben tener cuidado en el ofrecimiento de productos financieros. El diseño de un producto equivocado puede conducir al desastre financiero de un cliente al no coincidir con sus necesidades. Estos préstamos pueden ser tan grandes que pueden llevar al sobreendeudamiento: en una etapa temprana, el cliente puede tener mayor voluntad y capacidad de pago; sin embargo, con el paso del tiempo, puede tener más dificultades. El sobreendeudamiento, realizado muchas veces por las mismas entidades que otorgan créditos a un mismo cliente (ya sea como una nueva institución u otorgándole un crédito en paralelo al ya vigente), puede conducir a que los deudores no puedan pagar. Así, se genera una posible morosidad, que tiene un impacto en las provisiones de la institución y, por ende, afecta la rentabilidad.
En esa línea, la SBS aprobó la Resolución SBS 6941 (Reglamento para la Administración del Riesgo de Sobreendeudamiento de los Deudores Minoristas), en la que establece «sobreendeudamiento» como el «nivel de endeudamiento en el sistema financiero que, por su carácter excesivo respecto de los ingresos y de la capacidad de pago pone en riesgo el repago de las obligaciones de un deudor minorista» (art. 2). Además, dispone que las empresas deberán establecer en sus políticas medidas que incorporen el riesgo crediticio; una de las variables diferenciadoras del riesgo es el número de instituciones con las que los deudores minoristas hayan contraído obligaciones. Por su parte, Cermeño, León y Mantilla (2011) establecen una relación directa de la morosidad con el sobreendeudamiento, por lo que el número de instituciones financieras es una de las variables. Para efectos del presente documento, se considera que un cliente se encuentra sobreendeudado cuando trabaja de manera simultánea con más de tres entidades financieras. Se considera este número como el estimado de acuerdo con políticas crediticias; además, será validado en entrevistas a expertos.

En un artículo propio publicado en una revista indexada brasilera, Toledo (2018) menciona que el sobreendeudamiento sucede en muchos casos cuando el cliente llega a tener deudas de forma simultánea con diversas entidades microfinancieras. Desde la propia experiencia, se han observado deudores que trabajan hasta con trece entidades financieras al mismo tiempo. Esta situación se origina muchas veces por la sobreoferta de préstamos de las instituciones y por el objetivo de incrementar el número de clientes. Además, al afrontar el cumplimiento de metas elevadas, muchos supervisores de los analistas o asesores de crédito tienden a otorgar préstamos con una evaluación más flexible, con mayores líneas de crédito o reduciendo las tasas de interés. En otras palabras, los préstamos 
pueden llegar a darse como una práctica insana. Finalmente, como afirman Rayo, Rodríguez y Lara (2011), hay mucho trabajo por hacer con respecto a revisiones futuras sobre incidencias de variables internas y externas que influyen en el incumplimiento de pagos. El presente documento pretende contribuir con ello.

\section{Metodología}

La presente investigación tiene un enfoque cuantitativo, puesto que «usa la recolección de datos para probar el postulado propuesto, con base en la medición numérica y el análisis estadístico, para establecer patrones de comportamiento y probar teorías» (Hernández, Fernández, \& Baptista, 2010, p. 4). Tiene un alcance correlacional y explicativo, porque, a través de una metodología estadística, se pretende demostrar la relación entre las variables de estudio. Asimismo, presenta un diseño no experimental con un horizonte de tiempo transversal, puesto que la metodología aplicada desarrollará las variables tal como ocurre en su contexto natural.

Se tiene como población de estudio al total de CMAC que operan en el Perú. Estas cajas, al igual que todas las instituciones del sistema financiero peruano, remiten información financiera a la SBS. Esta institución consolida toda la información y la publica en su página oficial para conocimiento de toda la población bajo ciertos formatos elaborados. Para el presente trabajo, se cuenta con la información de 48 meses (enero 2016-diciembre 2019). Además, en la investigación se realizaron entrevistas a profundidad a las personas especialistas en las diferentes áreas de negocios y riesgos, cuya participación en las instituciones microfinancieras son o fueron pertinentes para el caso del estudio planteado. Los datos recolectados fueron almacenados en una tabla de una base de datos relacional SQL ${ }^{2}$. Después de este proceso de almacenamiento, la información fue trasladada al aplicativo SPSS $^{3}$ Statistics para la construcción del análisis estadístico. A continuación, para el procesamiento y demostración de la incidencia del sobreendeudamiento en el riesgo de crédito de las CMAC, se presenta la operacionalización de las variables a utilizar y sus respectivos indicadores en la tabla 1.

Tabla 1. Operacionalización de variables

\begin{tabular}{|l|l|l|}
\hline \multicolumn{1}{|c|}{ Variables } & Denominación & \multicolumn{1}{c|}{ Indicadores } \\
\hline $\begin{array}{l}\text { Dependiente } \\
\text { (Y) }\end{array}$ & $\begin{array}{l}\text { Riesgo de } \\
\text { crédito (cartera } \\
\text { de alto riesgo) }\end{array}$ & $\begin{array}{l}\text { Y. Cartera vencida + cartera } \\
\text { refinanciada / reestructurada } \\
\text { + cartera judicial / total de } \\
\text { saldo de cartera }\end{array}$ \\
\hline $\begin{array}{l}\text { Indepen- } \\
\text { diente }(\mathrm{X})\end{array}$ & $\begin{array}{l}\text { Sobreendeu- } \\
\text { damiento de } \\
\text { clientes }\end{array}$ & $\begin{array}{l}\mathrm{X}_{1} \text {. Número de deudores con } \\
\text { más de tres entidades finan- } \\
\text { cieras / total de deudores }\end{array}$ \\
\cline { 3 - 3 } & $\begin{array}{l}\mathrm{X}_{2} \text {. Cartera de deudores con } \\
\text { más de tres entidades finan- } \\
\text { cieras / total de cartera }\end{array}$ \\
\hline
\end{tabular}

\section{Procesamiento y análisis de resultados}

Para el desarrollo del presente trabajo, se utilizará el modelo estadístico Cross Industry Standard Process for Data Mining (CRISP-DM), que es una metodología que, a través de una secuencia de pasos, trabaja con información de una minería de datos, también conocida como data mining. Se trata de una de las metodologías más usadas en la actualidad. Identificar qué variables pueden influir en otras es un aspecto importante, que corresponde al uso de técnicas estadísticas. El sector de las microfinanzas es un negocio a gran escala. En ese sentido, es necesaria la utilización

2 SQL: Structured Query Language. Programa utilizado para diseñar y recuperar información de bases de datos relacionales

3 SPSS: Statiscal Package for the Social Sciences 
de herramientas estadísticas que manejen el uso del data mining. Para Pérez y Santín (2007) es fundamental usar data mining, dado que estas herramientas «tienen como objetivo descubrir patrones, perfiles y tendencias a través del análisis de estos datos» (p. 1). Con respecto a la secuencia de pasos que sigue esta metodología, se encuentran los puntos que se comentarán a continuación.

\subsection{Comprensión del negocio}

Esta investigación se ha centrado en el grupo de entidades microfinancieras de las CMAC, que tienen el $50 \%$ de participación en el sector. En las CMAC se han observado problemas relacionados al deterioro de su cartera, que afectan directamente sus resultados económico-financieros a partir del incremento de mayores provisiones. Tal problemática ha sido descrita a lo largo de la investigación desde varios puntos de vista. Esta investigación se centra en determinar la relación de la variable independiente, sobreendeudamiento de clientes; y el riesgo de crédito de las CMAC.

\subsection{Análisis de los datos}

Los datos recolectados para esta variable han sido obtenidos del reporte crediticio de deudores (RCD). Para la construcción de este indicador, se consideraron todos los deudores y el respectivo saldo que tienen en más de tres entidades financieras de forma paralela, a partir del total de cartera de cada CMAC. El objetivo que se busca es demostrar que los clientes que trabajan con más de tres entidades, definidos en la presente investigación como «sobreendeudados», están relacionados directamente con el riesgo de crédito. Esto se validará con las entrevistas a expertos.

En la figura 1, se aprecia la evolución del saldo de la cartera de todos los deudores que tienen créditos en más de tres instituciones. Se observa que aproximadamente el promedio de cartera de este indicador se encuentra entre el 20\% y 22\%. Asimismo, la figura muestra el comportamiento de la cartera de alto riesgo. De acuerdo con los datos, solo se podría decir que el saldo de la cartera de los deudores que trabajan con más de tres entidades ha tenido una paulatina

Figura 1. Sobreendeudamiento y cartera de alto riesgo (riesgo de crédito)

Cartera de deudores con 4 entidades o más y Cartera de alto riesgo

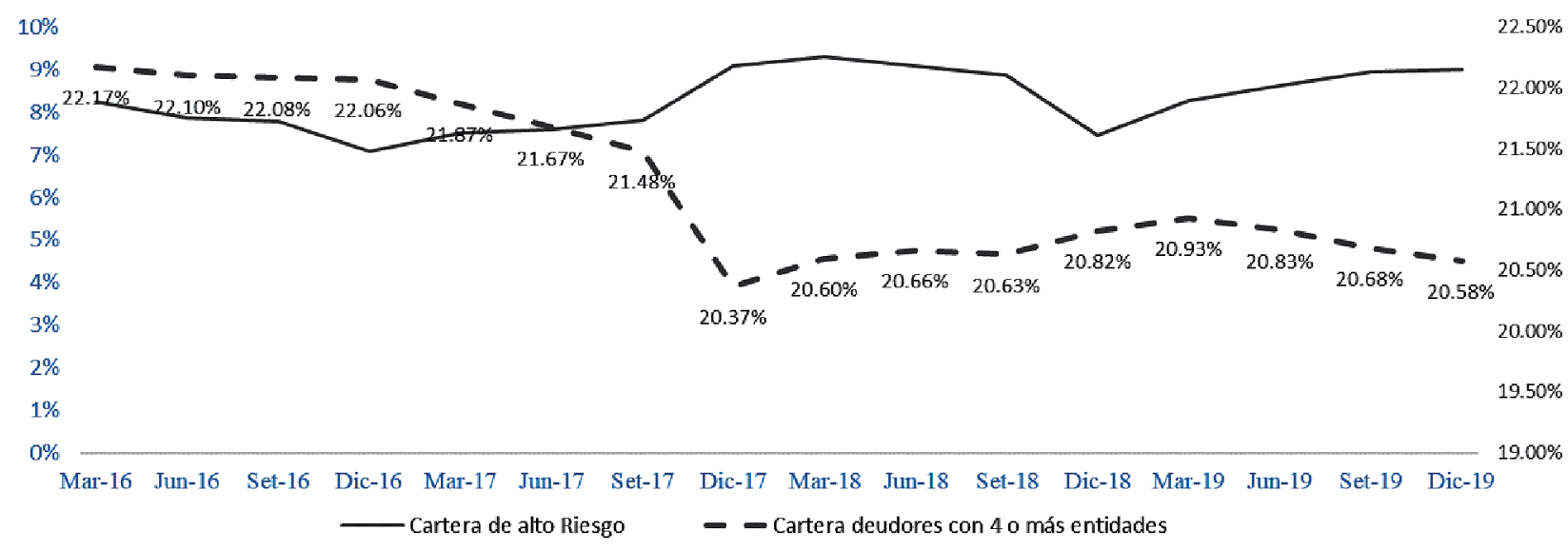


reducción en los últimos años. Cabe señalar que el comportamiento de la variable morosidad se ve influenciado por diversos factores y no solo por el sobreendeudamiento. Se podrá apreciar la relación de esta variable cuando se efectúe el análisis estadístico y se conozca su relación con el riesgo de crédito.

\subsection{Exploración de datos}

El análisis estadístico es básico en la investigación. En este caso, los estadísticos de medias, medianas y moda permiten analizar la característica de los datos, y explorar si estos presentan indicios de una distribución normal ${ }^{4}$. Es característico de una distribución normal que presente valores de media, mediana y moda similares. En la tabla de estadísticos descriptivos de las variables, se observa que las variables analizadas presentan, mayormente, medias, mediana y modas muy dispersas (ver tabla 2). Por lo tanto, no cumplirían con la característica de normalidad. Para determinar el tipo de distribución estadística, se aplicará la prueba de Kolmogorov-Smirnov.

En los estadísticos calculados, también se ha incluido el indicador de asimetría ${ }^{5}$, que permite conocer la forma de la distribución de las variables. El estadístico de asimetría indica si los datos están distribuidos de manera uniforme respecto de la media. Una característica de normalidad de los datos tendrá como resultado un estadístico de asimetría lo más cercano a cero $(+/-0,5)$. En cambio, un indicador positivo $(>+0,5)$ indica que los datos se encuentran distribuidos por encima de la media; y un indicador negativo $(<-0,5)$, que se encuentran por debajo. En la tabla 2, se presen- tan los estadísticos descriptivos de las variables, tanto dependientes como independientes. Así, se observa que existe una marcada diferencia entre los estadísticos de media, mediana y moda. Asimismo, el estadístico de asimetría sugiere una distribución no normal, pues se encuentran por encima de los $(+/-0,5)$.

Tabla 2. Estadísticos descriptivos de las variables

\begin{tabular}{|l|c|c|c|c|c|}
\hline & Media & Mediana & Moda & $\begin{array}{c}\text { Desvia- } \\
\text { ción }\end{array}$ & $\begin{array}{c}\text { Asime- } \\
\text { tría }\end{array}$ \\
\hline $\begin{array}{l}\text { Riesgo de cré- } \\
\text { dito (Y) }\end{array}$ & 11,6 & 9,1 & 3,6 & 6,2 & 0,94 \\
\hline $\begin{array}{l}\text { Deudores de } \\
\text { más de cuatro } \\
\text { entidades }\end{array}$ & 21.219 & 12.710 & 3904 & 16.603 & 0,75 \\
\hline $\begin{array}{l}\text { Saldo de más de } \\
\text { cuatro entidades }\end{array}$ & 338.583 & 3904 & 23.445 & 282.831 & 0,67 \\
\hline
\end{tabular}

Cabe indicar que es de mucha importancia para el análisis si existe o no normalidad en las variables que se presentan. Para ello, con el objetivo de complementar el análisis de estadísticos descriptivos y confirmar los indicios de no normalidad de las variables, se utilizará la prueba de Kolmogorov-Smirnov. Esta es una prueba no paramétrica que pertenece al grupo de pruebas llamadas «de bondad de ajuste» y tiene como objetivo indicar si los datos presentan una distribución especifica definida por el investigador. Con respecto a la prueba de Kolmogorov-Smirnov, Romero (2016) señala que «es una prueba de significación estadística para verificar si los datos de la muestra proceden de una distribución normal. Se emplea para variables cuantitativas y cuando el tamaño muestral es mayor de 50» (p. 36). De acuerdo con la teoría respectiva,

4 Es muy importante la determinación de la normalidad de los datos, pues permitirá decidir qué tipo de prueba se puede llevar a cabo: pruebas paramétricas o no paramétricas.

5 La asimetría es la medida que indica la simetría de la distribución de una variable en referencia a la media aritmética; puede ser positiva o negativa. 
para aceptar la hipótesis nula, el valor de la significancia estadística (valor p) debe ser mayor a 0,05. Por lo tanto, desde la investigación, se establecen las siguientes premisas:

- $\mathrm{H}_{0:}$ Hipótesis nula: el conjunto de datos sigue una distribución normal.

- $\mathrm{H}_{1:}$ Hipótesis alternativa: el conjunto de datos no sigue una distribución normal.

La tabla 3 muestra el estadístico de prueba y el valor p (significancia asintótica) para cada una de las variables. Se observa que todos los valores de p son menores a 0,05 . Por lo tanto, se rechaza la hipótesis nula («el conjunto de datos no sigue una distribución normal»); es decir, se rechaza la homogeneidad, y se afirma que hay diferencias en la distribución de las variables analizadas y la distribución normal. Entonces, no hay homogeneidad y existe evidencia suficiente para concluir que los datos de las variables no presentan distribución normal con un nivel de confianza de 95\%.

\section{Tabla 3. Normalidad de las variables: prueba de Kolmogorov-Smirnov}

\begin{tabular}{|l|c|c|c|c|}
\hline \multirow{2}{*}{ Variables } & \multicolumn{4}{|c|}{ Prueba de Kolmogorov-Smirnov } \\
\cline { 2 - 5 } & $\begin{array}{c}\text { Estadís- } \\
\text { tico de } \\
\text { prueba } \\
\mathbf{z}\end{array}$ & $\begin{array}{c}\text { Significación } \\
\text { estadística. } \\
\text { asintótica } \\
\text { valor } \mathbf{p}\end{array}$ & Media & $\begin{array}{c}\text { Desviación } \\
\text { típica }\end{array}$ \\
\hline $\begin{array}{l}\text { Cartera de alto } \\
\text { riesgo }\end{array}$ & 0,211 &, 000 & 11,62 & 6,19761 \\
\hline $\begin{array}{l}\text { Ratio número } \\
\text { de deudores con } \\
\text { cuatro entidades }\end{array}$ & 0,197 &, 000 & 0,175 & 0,06286 \\
\hline $\begin{array}{l}\text { Ratio saldo de } \\
\text { deudores con } \\
\text { cuatro entidades }\end{array}$ & 0,138 &, 000 & 0,229 & 0,05529 \\
\hline
\end{tabular}

Fuente: Elaboración propia, adaptado del SPSS.

\subsection{Modelamiento}

El objetivo principal de esta etapa es presentar la técnica estadística apropiada que permita identificar el nivel de relación de cada una de las variables analizadas. Además, debe permitir determinar cuáles son las variables que tienen mayor relevancia sobre la variable dependiente para las CMAC. Para este trabajo, se utilizó la técnica regresión logística. Un criterio importante para la selección de dicha técnica es que se ajusta al tipo de variables cuantitativas construidas en la etapa de preparación de datos, así como a las características y comportamiento de las variables, como, por ejemplo, la distribución no normal también llamada «distribución no paramétrica». Ambos aspectos fueron analizados y demostrados en los estadísticos de las variables y prueba de Kolmogorov-Smirnov, comentados anteriormente.

A continuación, se mostrarán los resultados de salida del análisis cuando se ejecuta la regresión logística. Se expondrán los resultados en función al objetivo planteado en esta investigación. El resultado está centrado en el nivel de significación (valor - p); y el tipo de relación o comportamiento, que puede ser positivo o negativo, sobre la base del signo del coeficiente B y el grado de fuerza de la relación que presenta la regresión logística, es decir, $\operatorname{Exp}(B)$. Para el análisis, se ha considerado la siguiente interpretación de los indicadores ${ }^{6}$ :

- El coeficiente del parámetro (B) es el signo del coeficiente que indicará el tipo de relación con la variable dependiente. Si es positiva, va en la misma dirección; si es negativa, va en sentido contrario. Cabe indicar que los valores de los coeficientes no tienen interpretación: solo sirven para ver la relación positiva o negativa con respecto a la variable dependiente.

6 La información ha sido adaptada de Cárdenas (2014, 2015). 
- El $\operatorname{Exp}(\mathrm{B})$, también llamado odd ratio, es una medida de asociación entre variables utilizadas en modelos de regresión logística. Indicará la fortaleza de la relación con la variable dependiente. Este indicador es la razón por que se usa la regresión logística.

- El nivel de significancia (valor - p) explica si la variable independiente es significativa o no. Si el valor es menor a 0,05 , indica que el modelo explica la relación con la variable dependiente. Las que son mayores a 0,05 no son significativas.

Considerando los criterios indicados, el análisis permite presentar los siguientes resultados:

Tabla 4. Resultados del modelamiento

\begin{tabular}{|l|c|c|c|}
\hline $\begin{array}{c}\text { Variable sobreendeudamiento } \\
\text { y su relación con la variable } \\
\text { dependiente }\end{array}$ & B & $\operatorname{Exp}(B)$ & $\begin{array}{c}\text { Significancia } \\
\text { (Valor - p) }\end{array}$ \\
\hline $\begin{array}{l}\text { Ratios número de deudores } \\
\text { con cuatro o más entidades }\end{array}$ & 2,104 & 8,198 & 0,000 \\
\hline $\begin{array}{l}\text { Ratio saldo de deudores con } \\
\text { cuatro o más entidades }\end{array}$ & 1,129 & 7,247 & 0,000 \\
\hline
\end{tabular}

Fuente: Elaboración propia, adaptado del SPSS.

- Coeficiente B (positivo) en ambos casos: a mayor sobreendeudamiento (si saldos y clientes aumentan en número de entidades), crece la probabilidad de incrementar la cartera de alto riesgo.

- $\operatorname{Exp}(B)$ : indica que la fortaleza de la variable es de 8,198 y 7,247 el número de veces con respecto a la variable explicada; es decir, por cada incremento en la unidad de los saldos o clientes con cuatro o más entidades financieras, existe una alta probabilidad de que los indicadores del riesgo de crédito se incrementen.

- Significancia (valor - p) menor a 0,05: indica que el modelo explica el evento; es decir la variable sobreendeudamiento con sus indicadores, ratio número y saldo de deudores con cuatro o más entidades explica la variable dependiente.

El presente trabajo se ha complementado con entrevistas a profundidad realizadas a expertos en el negocio microfinanciero, a través de preguntas estructuradas. De acuerdo al análisis realizado en estas entrevistas y luego de procesar dicha información, se puede concluir que, a partir de la opinión de los entrevistados, la variable sobreendeudamiento, medida como el número de deudores y saldo de clientes con cuatro o más entidades, es una variable que explica el riesgo de crédito. Los expertos consideran que estas instituciones se disputan al mismo cliente y existe poca bancarización; además, los clientes no usan los mayores volúmenes de dinero en sus negocios, lo que origina problemas de liquidez en un corto o mediano plazo para el pago de sus deudas. Esto va alineado con lo presentado en el marco teórico, en el que se sostiene que diversos autores señalan que el sobreendeudamiento es un grave riesgo para la industria microfinanciera.

Tabla 5. Resultado de entrevistas a especialistas en microfinanzas

\begin{tabular}{|l|l|}
\hline $\begin{array}{l}\text { Resultados de } \\
\text { entrevistas }\end{array}$ & \multicolumn{1}{c|}{\begin{tabular}{c}
\multicolumn{1}{c|}{ Palabras claves o identificación de temas } \\
comunes
\end{tabular}} \\
\hline $\begin{array}{l}\text { Acerca del } \\
\text { sobreendeu- } \\
\text { damiento }\end{array}$ & $\begin{array}{l}\text { Definitivamente una variable que podría expli- } \\
\text { car la cartera de alto riesgo / considera como } \\
\text { indicador al número de entidades / poca ban- } \\
\text { carización / peleas por tener al mismo cliente } \\
\text { / cumplir metas / competir con prestamistas } \\
\text { informales / mala inversión del dinero }\end{array}$ \\
\hline
\end{tabular}

A lo largo de la investigación, se ha hablado sobre el crecimiento del negocio de manera acelerada, lo que ha originado mayor competitividad, disminución de las tasas de interés, relajamiento de políticas, e incremento de metas de los funcionarios o asesores de negocios que han llegado a la necesidad de llevar a sus

Contabilidad y Negocios (16) 32, 2021 / ISSN 1992-1896 
clientes al sobreendeudamiento. El resultado del estudio es un aporte para las decisiones estratégicas de las CMAC. Se ha evidenciado que, a mayor número de entidades financieras y saldo de cartera que tenga un deudor, mayores serán las dificultades de pago, ya sea por una insuficiente capacidad de pago o por la falta de voluntad por cumplir con las obligaciones financieras. De esta manera, el incremento de riesgo se ve directamente impactado.

\section{Conclusiones y recomendaciones}

Las CMAC en Perú son un grupo de instituciones financieras especializadas en la atención de la micro y pequeña empresa en el país. Representan aproximadamente el 50\% del mercado microfinanciero y su participación en la actividad económica es notable, pues cumplen el rol fundamental de brindar acceso e inclusión al financiamiento de los micro y pequeños empresarios. Sin embargo, dicho dinamismo va de la mano con la problemática referida al incremento del riesgo de crédito (entendida como la cartera de alto riesgo) de este importante grupo de instituciones microfinancieras.

Sobre la base de lo planteado en esta investigación, se puede afirmar que el sobreendeudamiento es un factor de riesgo importante para las CMAC. Esto se determinó a través de los resultados del análisis estadístico desarrollado, utilizando la metodología CRISP-DM y el uso de regresión logística, trabajadas con data mining obtenidos de fuentes secundarias. Los resultados fueron contrastados con entrevistas a expertos en el sector microfinanciero, quienes validaron en su conjunto que la variable sobreendeudamiento explica el riesgo de crédito.

En síntesis, se pudo determinar que el sobreendeudamiento de clientes, medido como el número y saldo de deudores con cuatro o más entidades, influye en forma directa y positiva en el riesgo de crédito de las CMAC. Sobre todo, como resultado del análisis realizado, se determinó que dicha variable tiene una gran fortaleza de relación con la variable dependiente. En opinión de los investigadores y de los resultados de las entrevistas a expertos, esto se relaciona con la sobreoferta de productos financieros que ofrecen las CMAC a los clientes. $\mathrm{Al}$ no tener cultura financiera, los clientes comienzan a tomar dinero de varias entidades de forma paralela, por lo que sus flujos futuros no coinciden con el efectivo que necesitan para hacer frente a sus pagos. Las estrategias que deben tener las CMAC deben estar orientadas a la reunificación de deudas, es decir, deben permitir agrupar todas las deudas en un único producto y con un único pago. Adicionalmente, deben realizar seguimiento a los clientes de forma constante para observar su comportamiento con respecto al número de entidades con las que pueden relacionarse en el tiempo.

Entonces, las estrategias a considerar deben estar dirigidas al control de la compra de deudas. Tal acción se realiza de forma agresiva en este mercado, en el que prácticamente los asesores se están "canibalizando» al arrebatarse a los mismos clientes. Las CMAC deben tener en cuenta que la insolvencia del cliente no se verá al momento de otorgar el crédito, sino que se verá reflejada más adelante.

\section{Referencias}

Aguilar, G., Camargo, G., \& Morales, R. (2004). Análisis de la morosidad en el sistema bancario peruano. Informe final de investigación. Instituto de Estudios Peruanos, 1, 1-108.

Alcántara, E., \& Damián, J. (2020). Causas generadoras de la morosidad crediticia y el grado de afectación en el mercado financiero, en el distrito de Chiclayo, periodo 2012-2013 (Tesis de maestría, Escuela de Posgrado, Universidad Nacional Pedro Ruiz Gallo. Lambayeque, Perú). 
Cárdenas, J. (2014, 21 de febrero). Qué es la regresión logística binaria y cómo analizarla en seis pasos. Networkianos. Blog de Sociología. Recuperado de http:// networkianos.com/regresion-logistica-binaria/ [Consulta: 8 de mayo 2020].

Cárdenas, J. (2015, 1 de diciembre). Odd ratio: qué es y cómo se interpreta. Networkianos. Blog de Sociología. Recuperado de http://networkianos.com/odd-ratioque-es-como-se-interpreta/\#toc-2 [Consulta: 11 de julio 2020].

Cermeño, R., León, J., \& Mantilla, G. (2011). Determinantes de la morosidad: un estudio panel para el uso de las cajas municipales de ahorro y crédito del Perú, 20032010 (Documento de trabajo 513). México, D. F.: Centro de Investigación y Docencia Económicas.

Congreso de la República. (2003, 3 de julio). Ley de Promoción y Formalización de la Micro y Pequeña Empresa [Ley 28015]. Recuperado de http://www.sunat.gob. pe/orientacion/mypes/normas/ley-28015.pdf [Consulta:15 de junio 2020].

García, E. (2018, 11 de noviembre). Se desata agresiva compra de deuda en campaña navideña. Diario Gestión. Recuperado de https://gestion.pe/economia/desataagresiva-compra-deuda-campana-navidena-249558noticia/?ref=gesr [Consulta:12 de junio 2020].

Goldberg, M., \& Palladini, E. (2011). Gestión de riesgos y creación de valor con las microfinanzas. Washington, D. C.: The International Bank for Reconstruction and Development/The World Bank.

González, A., \& González-Vega, C. (2003). Sobreendeudamiento en las microfinanzas bolivianas, 1997-2001. Ohio: USAID

Hernández, R., Fernández, C., \& Baptista, P. (2010). Metodología de la investigación (5ta ed.). México, D. F.: MacGraw-Hill.

Ledgerwood, J. (2000). Manual de las microfinanzas: una perspectiva institucional y financiera. Washington, D. C.: Banco Mundial.
Ministerio de la Producción. (2020). Estadistica Mipyme. Recuperado de https://ogeiee.produce.gob.pe/index. php/en/shortcode/estadistica-oee/estadisticas-mipyme [Consulta: 01 de junio 2020].

Pereira, C. (2019). Actualidad de la gestión empresarial en las pymes (Current Business Management in SMEs). Apuntes Contables, 24, 39-53. https://doi. org/10.18601/16577175.n24.03

Pérez, C., \& Santín, D. (2007). Minería de datos: técnicas y herramientas. Madrid: Paraninfo.

Rayo, S., Rodríguez, M., \& Lara, J. (2011). Un caso empírico en la evaluación del riesgo de crédito de una institución de microfinanzas peruana. Contabilidad y Negocios, 6(11), 21-30.

Romero, M. (2016). Pruebas de bondad de ajuste a una distribución normal. Revista Enfermería Del Trabajo, 6(3), 105-114. Recuperado de https://dialnet.unirioja.es/servlet/articulo?codigo=5633043 [Consulta: 16 de mayo 2020].

Rossi, J. (2017). Regulación del endeudamiento y sobreendeudamiento del consumidor en la República Argentina. Morón: Universidad de Morón.

Schicks, J. (2010). Microfinance Over-Indebtedness: Understanding its drivers and challenging the common myths (Business, CEB Working Paper 10-048). Bruselas: Universite Libre de Bruxelles.

Superintendencia de Banca, Seguros y AFP (SBS). (2008a, 25 de agosto). Aprueban Medidas para Reducir el Sobreendeudamiento por Créditos de Consumo en el Sistema Financiero [Resolucion SBS No 6941-2008].

Superintendencia de Banca, Seguros y AFP (SBS). (2008b, 19 de noviembre). Se Aprueba el Nuevo Reglamento para la Evaluación y Clasificación del Deudor y la Exigencia de Provisiones [Resolucion SBS No 11356-2008].

Superintendencia de Banca y Seguro (SBS). (2019, 1 de febrero). Texto Concordado de la Ley General del Sistema Financiero y del Sistema de Seguros y Orgánica de la Superintendencia de Banca y Seguros [Ley 26702].

Contabilidad y Negocios (16) 32, 2021 / ISSN 1992-1896 
Toledo, E. (2018). Microfinanzas en el Perú y los desafíos de la bancarización. REMIPE-Revista de Micro e Pequenas Empresas e Empreendedorismo Da Fatec Osasco, 4(1 jan-jun), 48-62. https://doi.org/10.21574/ remipe.v4i1.111

Vela, L., Uriol, J., Medina, O., Palacios, F., \& Pintado, E. (2012). Los factores que determinan la calidad de la cartera crediticia de las entidades microfinancieras de la Amazonía peruana en el periodo 2008-2011.
Universidad Nacional Pedro Ruiz Gallo. Recuperado de https://web.ua.es/es/giecryal/documentos/microfinanzas-amazonia.pdf [Consulta: 11 de junio 2020].

Fecha de recepción: 07 de marzo de 2021 Fecha de aceptación: 08 de julio de 2021 Correspondencia: toledo.ej@pucp.edu.pe victor.leon2@unmsm.edu.pe 